\title{
Cross-polarization dynamics and conformational study of variously sized cellulose crystallites using solid-state ${ }^{13} \mathrm{C}$ NMR
}

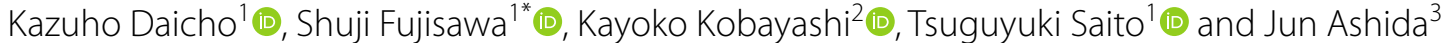

\begin{abstract}
Cellulose forms crystalline fibrils, via biosynthesis, that can be just a few nanometers wide. The crystallinity is a structural factor related to material performance. Recently, many routes to isolate these fibrils as nanocellulose have been developed, and there exist various types of nanocellulose with different crystallinities. Quantitative assessment of the crystallinity of nanocellulose is thus essential to advance knowledge in the high performance and functionality of such materials. Solid-state ${ }^{13} \mathrm{C}$ cross-polarization/magic-angle spinning (CP/MAS) nuclear magnetic resonance (NMR) spectroscopy is a strong tool to investigate the structural features and dynamics of solid cellulose. The crystallinity is often evaluated by using the NMR signal ratio of the C4 crystalline and noncrystalline regions as a crystallinity index (CI) value. To calculate the $\mathrm{Cl}$ value, it is necessary to examine the dependence of the contact time (CT) for CP on the signal intensity and set the optimum CT at a maximum of the signal intensity. However, the dependence has not been investigated for evaluation of the $\mathrm{Cl}$ value of various cellulose samples with different crystal sizes. Here, we optimized the $\mathrm{CT}$ for evaluation of the $\mathrm{Cl}$ value of cellulose with different crystal sizes. The error in the $\mathrm{Cl}$ at the optimized $\mathrm{CT}$ was $\sim 3 \%$. At the optimized $\mathrm{CT}$, the structural change after surface modification by TEMPO-oxidation was also analyzed from the NMR spectra of the $\mathrm{C} 6$ region. The relationship between the $\mathrm{Cl}$ value and the degree of oxidation shows that it is possible to evaluate the degree of oxidation from the NMR spectra irrespective of the crystallinity of cellulose. Furthermore, the C4-based Cl value was linearly correlated with the C6-based trans-gauche (tg) ratio, which is approximated by a function, $\mathrm{Cl}=0.9$ tg ratio.
\end{abstract}

Keywords: ${ }^{13} \mathrm{C}$ CP/MAS NMR, Crystallinity, Nanocellulose, TEMPO-oxidation

\section{Introduction}

The crystallinity of cellulose is a structural factor related to material performance, such as to the dielectric constant, thermal conductivity, and modulus [1-3]. In biological structure, cellulose forms crystalline fibrils with a few nanometers wide and large specific surface areas, which are histologically defined as cellulose microfibril. Recently, many routes to isolate the microfibril or fine-bundled microfibril have been developed [4-6]. These crystalline fibrils are collectively known as

\footnotetext{
*Correspondence: afujisawa@mail.ecc.u-tokyo.ac.jp

${ }^{1}$ Graduate School of Agricultural and Life Sciences, The University

of Tokyo, Bunkyo-ku, Tokyo 113-8657, Japan

Full list of author information is available at the end of the article
}

"nanocellulose" and are attracting attention as an emerging sustainable material with high performance and functionality. Today, there exist various types of nanocellulose with different crystallinities. Quantitative assessment of these structural factors for nanocellulose is thus essential to ensure adequate understanding of its functionality.

Solid-state ${ }^{13} \mathrm{C}$ cross-polarization/magic-angle spinning (CP/MAS) nuclear magnetic resonance (NMR) spectroscopy provides information on the conformation of the constituent carbon atoms of a system. Use of this NMR technique promotes a greater understanding of the structural features and dynamics of solid cellulose [712]. In the NMR spectra, the $C 4$ and $C 6$ carbon atoms are distinguishable into crystalline and noncrystalline signals [13], which have been attributed to the atoms at the core 
and surface regions of a crystallite, respectively. In particular, the signal ratio of the $\mathrm{C} 4$ crystalline and noncrystalline phases has been used as the crystallinity index (CI) value of nanocellulose [14]; even for nanocelluloses with high crystallinities, the contribution of the surface chains to the CI value is significant, due to the high surface-tocore ratios. To calculate the $\mathrm{CI}$ value, it is necessary to examine the dependence of the contact time (CT) for $\mathrm{CP}$ on the signal intensity and set the optimum CT to maximize the signal intensity [14-16]. However, the dependence has not been well investigated for cellulose samples with different crystallinities, even though the crystallinity could influence the CP dynamics.

Herein, we optimized the CT for the evaluation of the CI value of cellulose with different crystallinities. The crystallinity of the cellulose samples was finely tuned by changing the crystal size from a few nanometers to tens of nanometers. The NMR spectra were recorded at various CTs $(0.1-10 \mathrm{~ms})$, and the dependency of the CT to the signal intensity of the $\mathrm{C} 4$ crystalline and noncrystalline phases was investigated. At an optimized CT, the structural change via surface modification of native cellulose by TEMPO-oxidation was also analyzed from the NMR spectra of the C6 region.

\section{Methods}

\section{Materials}

Five cellulosic samples were used as the starting materials: a softwood dissolving pulp (DP), two types of TEMPO-oxidized pulps with different carboxylate contents, cotton linters, and a mantle of tunicate Halocynthia roretzi. The DP and one TEMPO-oxidized pulp (carboxylate content: $1.2 \mathrm{mmol} / \mathrm{g}$ ) was supplied by Nippon Paper Industries Co. Ltd., (Tokyo, Japan). The other TEMPO-oxidized pulp (carboxylate content: $1.8 \mathrm{mmol} / \mathrm{g}$ ) was supplied by DKS Co. Ltd., Kyoto, Japan. The cotton linters were purchased from Advantec Toyo Co. Ltd., Tokyo, Japan. The tunicate mantle was supplied in an undried state by a domestic fish-processing company. Tunicate cellulose was purified from the mantle according to the procedure described in a previous report [17]. The cotton linters and tunicate cellulose $(0.1 \mathrm{~g}$ for each) were acid-hydrolyzed with a $2 \mathrm{M} \mathrm{HCl}$ solution at $105{ }^{\circ} \mathrm{C}$ for $4 \mathrm{~h}$. The residual solid hydrolysates were washed with distilled water by filtration. Amorphous cellulose was prepared from the cotton linters according to the method described in a previous report [18]. All the chemicals were of laboratory grade (FUJFILM Wako Pure Chemical Corporation, Osaka, Japan) and used as received.

\section{Sample preparation}

The DP $(1 \mathrm{~g})$ was TEMPO-oxidized with $\mathrm{NaClO}$ addition (10 mmol per gram of sample) according to the method described in a previous report [19]. The oxidized samples were treated with $\mathrm{NaBH}_{4}(0.1 \mathrm{~g})$ in water $(100 \mathrm{~mL})$ at $\mathrm{pH}$ 10 for $3 \mathrm{~h}$ to eliminate unstable aldehyde groups in the sample. The resulting pulp is from here on referred to as TEMPO-oxidized DP (TO-DP). The carboxylate content of TO-DP was determined to be $1.6 \mathrm{mmol} / \mathrm{g}$. Some of the $0.1 \% \mathrm{w} / \mathrm{w}$ suspension of the oxidized samples were mechanically treated by using a Microtec Physcotron NS-56 homogenizer (Microtec Co., Ltd. Chiba, Japan) equipped with a $20-\mathrm{mm}$ diameter shaft at $7500 \mathrm{rpm}$ for 6 min, followed by sonication using a Nihon Seiki US$300 \mathrm{~T}$ ultrasonic homogenizer equipped with a $26-\mathrm{mm}$ diameter tip at $70 \%$ output for $8 \mathrm{~min}$. The resulting sample is from here on referred to as TEMPO-oxidized cellulose nanofiber (TO-CNF). All the samples were freeze-dried from the wet state, containing $30 \% \mathrm{w} / \mathrm{w}$ tertbutyl alcohol, to prevent dry agglomeration [20].

\section{X-ray diffraction (XRD)}

The freeze-dried samples were conditioned at $23^{\circ} \mathrm{C}$ and $50 \%$ relative humidity for over 1 day before analysis. The conditioned samples were pressed at approximately $750 \mathrm{MPa}$ for $1 \mathrm{~min}$ using a tableting device for XRD. XRD measurements were performed by the reflection method with a diffraction angle $2 \theta$ range of $5-45^{\circ}$ using a Rigaku RINT 2000 diffractometer (RIGAKU Corporation, Tokyo, Japan) with Ni-filtered $\mathrm{Cu} \mathrm{K} \alpha$ radiation $(\lambda=0.1548 \mathrm{~nm})$ at $40 \mathrm{kV}$ and $40 \mathrm{~mA}$. The crystal size was calculated from the XRD peak corresponding to the ( $\left.\begin{array}{lll}2 & 0 & 0\end{array}\right)$ plane using the Scherrer equation (shape factor $K=0.9)$.

\section{NMR spectroscopy}

The samples conditioned at $23{ }^{\circ} \mathrm{C}$ and $50 \%$ relative humidity were packed into air-tight tubes and analyzed using CP/MAS ${ }^{13} \mathrm{C}$ NMR spectroscopy. The reason the dried sample was used for the analyses is described in a paper reported by Heux et al. [16]. The CP/MAS ${ }^{13} \mathrm{C}$ NMR measurements were performed using a JEOL JNM-ECAII 500 spectrometer (JEOL Ltd., Tokyo, Japan) equipped with a 3.2-mm HXMAS probe and $\mathrm{ZrO}_{2}$ rotors at $125.77 \mathrm{MHz}$ for ${ }^{13} \mathrm{C}$. The samples were spun at $15,000 \mathrm{~Hz}$ and the spectra were obtained with $0.1-10 \mathrm{~ms}$ $\mathrm{CT}$ and a 5 -s relaxation delay. The $\mathrm{CI}$ value was calculated from the integral ratio of the signals in the region of 93-86 and 86-80 ppm, corresponding to the crystalline and noncrystalline C4 carbon atoms of cellulose, respectively.

\section{Results and discussion C4-based $\mathrm{Cl}$ value}

The NMR spectra of the samples with different crystal sizes were recorded at various CTs $(0.1-10 \mathrm{~ms})$. The 
Scherrer crystal sizes of the acid hydrolysates of tunicin and cotton, DP, TO-DP, and TO-CNF were 10.0, 6.5, 4.0, 3.6, and $2.0 \mathrm{~nm}$, respectively. For all the samples, the intensity of the $\mathrm{C} 4$ region changed depending on the CT (Fig. 1a). Furthermore, the spectra became sharper and split into several peaks as the crystal size increased, which is clearly visible in the spectra of the tunicate cellulose. These results can be interpreted as the crystallographic inequivalence of the glycosidic unit, and the resolution is improved when the crystal size is larger [8]. In the case of the crystalline atoms in small crystallites or the noncrystalline atoms, the structural heterogeneity might be too large to produce clearly resolved peaks in the spectrum.

The dependency of the CT to the signal intensities of both the $\mathrm{C} 4$ crystalline and noncrystalline regions was then analyzed. Figure 1b, c shows the CP curves for the $\mathrm{C} 4$ crystalline and noncrystalline regions, respectively. The signal intensities were normalized at the maximum intensity for each carbon. In both regions, all the signal intensities were rapidly increased at the initial stage before reaching a maximum. The maximum of these intensities was at approximately 1-2 ms CT, irrespective of the crystal size. This region was in accordance with the reported CT as a preferable time for evaluation of the CI value of cellulosic materials [16, 21, 22].

At the initial increase in the $\mathrm{C} 4$ crystalline and noncrystalline regions, there was no significant difference in the trend and slope (Fig. 1b, c). The initial increase mainly reflects the efficiency of magnetization transfer from the ${ }^{1} \mathrm{H}$ spin reservoir to the ${ }^{13} \mathrm{C}$ spin, described by the time constant of $T_{\mathrm{CH}}$. In general, a rigid structure has a smaller $T_{\mathrm{CH}}$ than a nonrigid system, although the chemical structure may be the same. Therefore, this result indicates that the rigidity of the $\mathrm{C} 4$ carbons is similar in the time scale represented by the $T_{\mathrm{CH}}$ relaxation, regardless of the crystal size.

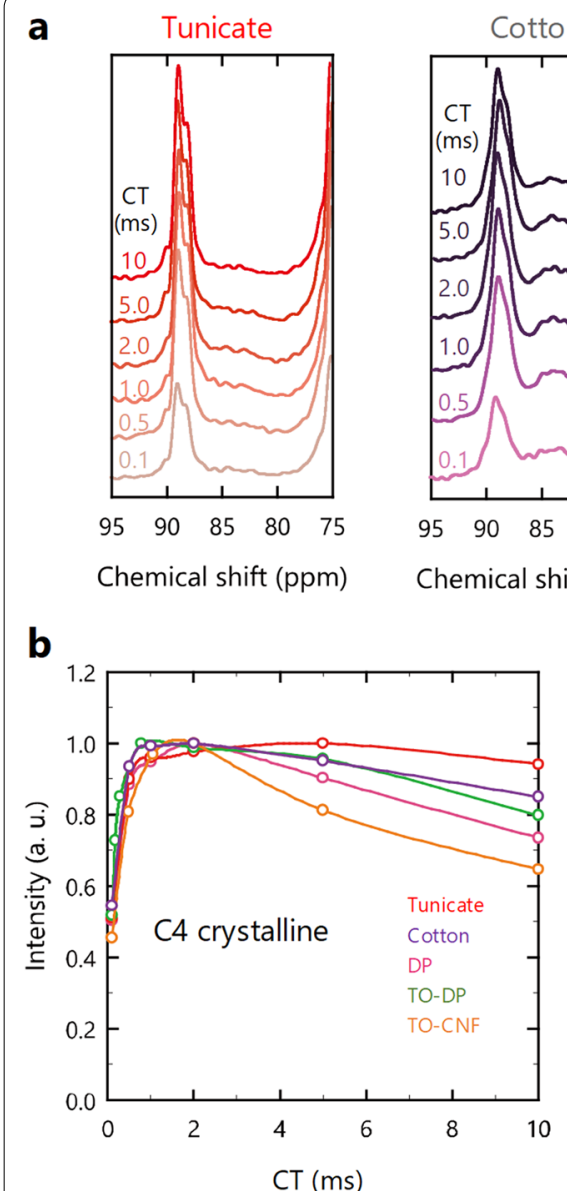

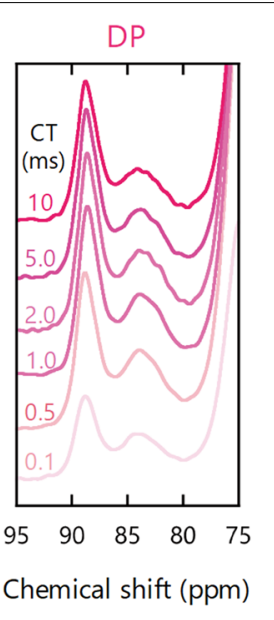

C

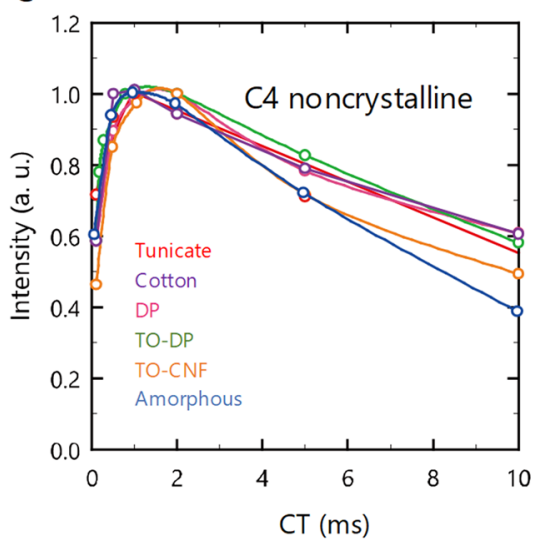

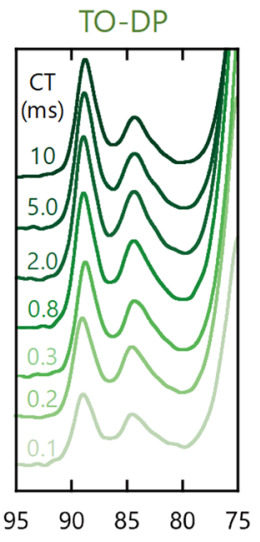

Chemical shift (ppm)

d

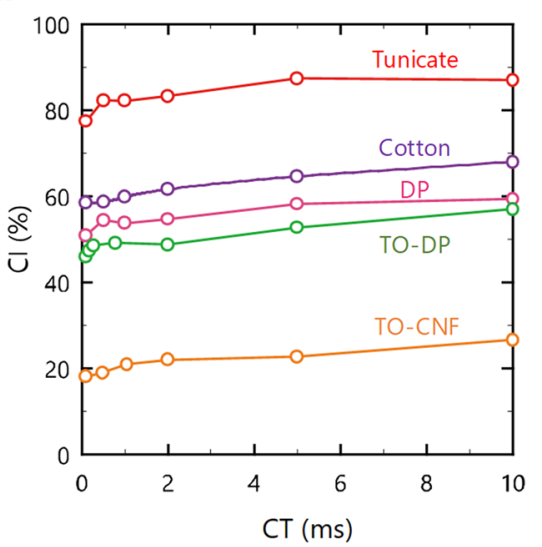

Fig. 1 a NMR spectra of a series of samples at several CTs. The Scherrer crystal sizes of the acid hydrolysates of tunicin and cotton, DP, TO-DP, and TO-CNF were $10,6.5,4.0,3.6$, and $2.0 \mathrm{~nm}$, respectively. b Dependency of CT on the signal intensity corresponding to the C4 crystalline region. $\mathbf{C}$ Dependency of $\mathrm{CT}$ on the signal intensity corresponding to the $\mathrm{C} 4$ noncrystalline region. $\mathbf{d}$ Dependency of $\mathrm{CT}$ on the $\mathrm{Cl}$ values 
In contrast, the decay in the $\mathrm{C} 4$ regions showed different behavior depending on the crystal size. The decay of each sample was steeper in the noncrystalline region than that in the crystalline region. In the crystalline region, the decay slope became steeper with a decrease in the crystal size (Fig. 1b). The decay at the longer CT reflects the relaxation of the ${ }^{1} \mathrm{H}$ and ${ }^{13} \mathrm{C}$ spins in the rotating frame $\left[T_{1 \rho}(\mathrm{H})\right.$ and $T_{1 \rho}(\mathrm{C})$, respectively], which are related to molecular mobility. Thus, the decay trends shown in Fig. 1b indicate that the crystalline region of large crystallites, such as the tunicate cellulose, have lower molecular mobility. However, the smaller crystallites exhibited the higher mobility, even in the crystalline region, because of the large contribution of the crystallite surface. Furthermore, the similar trend was visible in the decay of the noncrystalline region; the decay was faster with a decrease in the crystal size (Fig. 1c). Indeed, the conformational heterogeneity is present in both the crystalline and noncrystalline regions. It has been reported that part of the molecules that exist close to the surface sit in a noncrystalline conformation even in the interior of the microfibril [23, 24]. Our results also suggest this.

Another possibility of the existence of heterogeneity is also conceivable. It was previously found by some of us that the crystalline $\mathrm{C} 4$ signal decreased when the bundled fibers dispersed as microfibrils and, correspondingly, the noncrystalline signal increased [18]. Indeed, the cellulose molecules at the interface between bundled fibrils or at the surface of the microfibrils are partially crystallized. This interface has been regarded as an inaccessible surface, where solvent molecules cannot penetrate. It has been proposed that the NMR signal due to the molecules at the inaccessible surface appear in the noncrystalline region [25]. Taking these phenomena into account, some of the inaccessible molecules at the bundled interfaces are probably occupied in the crystalline region of the NMR spectra rather than in the noncrystalline region, thus causing the structural heterogeneity.

The CP curve of the amorphous cellulose sample, whose molecular sheet stacking and the atomic conformation are disordered and noncrystalline, respectively [26], was also compared with other cellulosic samples (Fig. 1c). The initial increase and the maximum were similar to that observed for the $\mathrm{C} 4$ noncrystalline carbons in other samples. The decay slope was slightly steeper than that of TO-CNF, but this difference is somewhat minimal. These results suggest that the glycoside linkage may be restricted even in the randomly packed cellulose molecules.

The CI values for all the samples were then plotted against the CT (Fig. 1d). In the range of approximately 1-2 ms CT, where the signal intensities were at a maximum, the $\mathrm{CI}$ values of each sample were almost the same, and the difference in the $\mathrm{CI}$ values was within only $3 \%$. At over $2 \mathrm{~ms} \mathrm{CT}$, the CI values gradually increased. This is because of the decrease in the intensity of the noncrystalline region resulting in overestimation of the CI value (see Fig. 1c). For a quantitative demonstration, the $\mathrm{CI}$ value of the mixture of the tunicate and amorphous cellulose was calculated from the NMR spectra recorded at $2 \mathrm{~ms} \mathrm{CT} \mathrm{(Fig.} \mathrm{2).} \mathrm{The} \mathrm{calculated} \mathrm{CI} \mathrm{value} \mathrm{was} 51 \%$, which is in good agreement with the ideal value (49\%) determined gravimetrically. These results indicate that, although the CP/MAS NMR spectroscopy is generally not quantitative for polymers having crystalline and amorphous regions, it is possible to evaluate the $\mathrm{CI}$ value of cellulose with an accuracy $<3 \%$ from the NMR spectra at the optimized CT of $2 \mathrm{~ms}$.

\section{Carboxylate groups at the $\mathrm{C} 6$}

In the process of nanocellulose production, cellulose is often surface modified in advance before wet disintegration. One example is by TEMPO-oxidation. By this method, the primary hydroxy groups exposed on the surface of the cellulose crystallite were converted to carboxyl groups, maintaining the crystallinity [27]. Figure 3a shows the NMR spectra of the C6 carbonyl region of TO-DP and TO-CNF at various CTs $(0.1-10 \mathrm{~ms})$. Both the shape and intensity of these NMR spectra changed depending on the $\mathrm{CT}$, similarly to the $\mathrm{C} 4$ region.

The signal intensities of the carbonyl carbon were then plotted against the CT, see Fig. 3b. Interestingly, TO-DP having bundled microfibrils showed similar CP dynamics with TO-CNF. The signal intensities of the carbonyl carbon increased more slowly than the signals of the $\mathrm{C} 4$

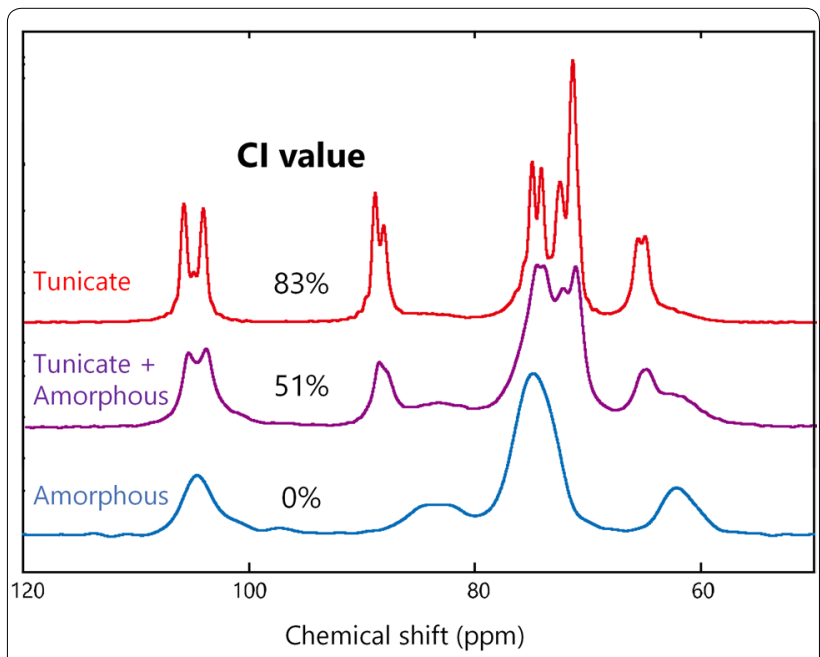

Fig. 2 NMR spectra of tunicate cellulose, a mixture of the tunicate and amorphous celluloses, and amorphous cellulose at $2 \mathrm{~ms}$ CT. The mass ratio of the tunicate cellulose in the mixture was $60 \%$ 

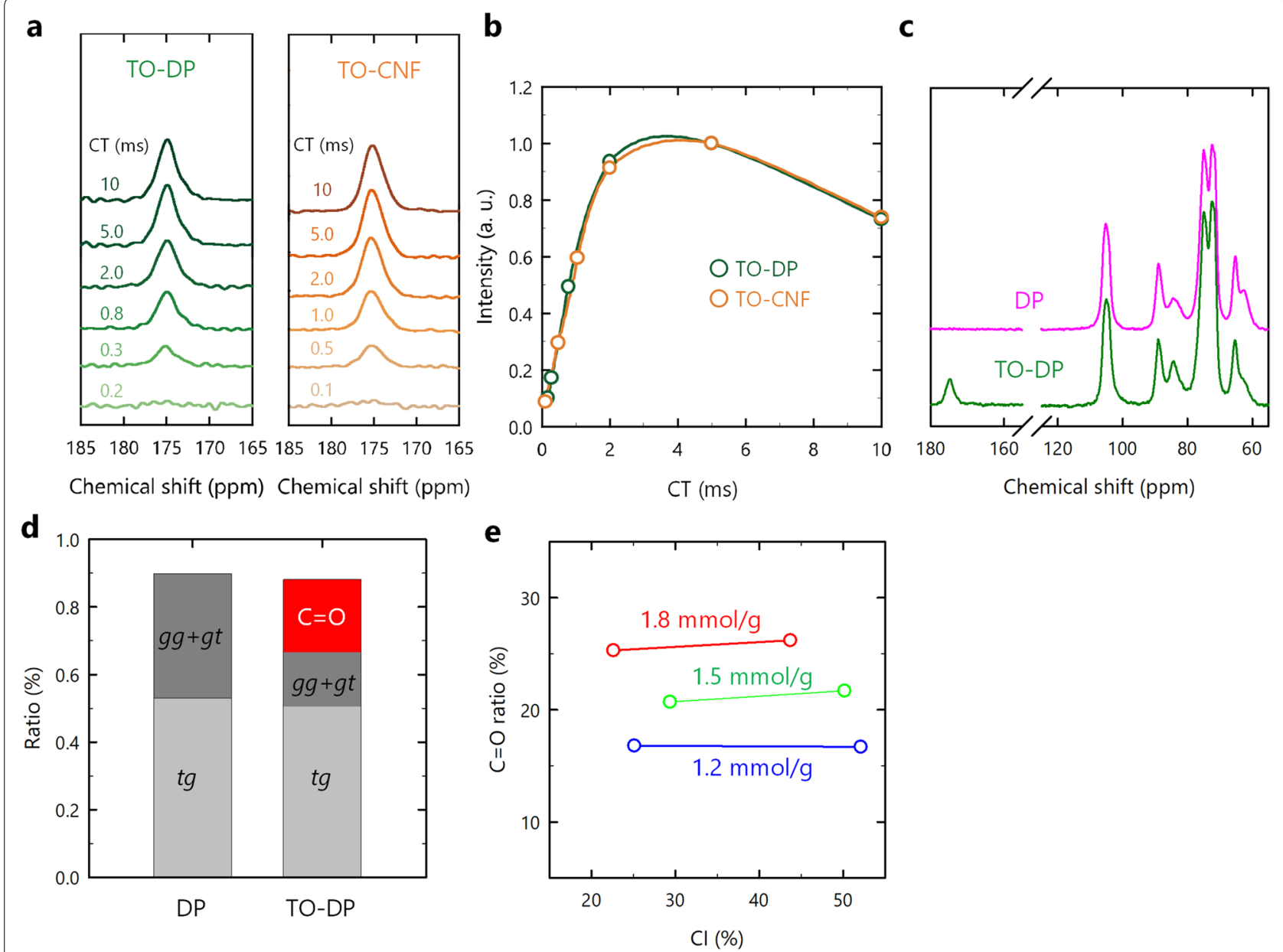

Fig. 3 a Spectral change in the carbonyl region of TO-DP and TO-CNF with CT. b Dependency of CT on the signal intensity corresponding to the carbonyl carbon. $\mathbf{c}$ Change in the NMR spectra at 2 ms CT with TEMPO-oxidation. $\mathbf{d}$ Change in the signal ratio of the conformation of the C6 hydroxy group and carbonyl carbon. e Relationship between the $\mathrm{Cl}$ value and the carbonyl ratio calculated as the signal ratio of the carbonyl carbon to the $\mathrm{C} 1$ carbon

carbon, and then reached a maximum at approximately 3 ms. In other words, the $T_{\mathrm{CH}}$ of the carbonyl carbon was larger than that of the C4 carbons (see Fig. 1 for the dependency of the CT to the signal intensity of the C4 carbons). This is likely because of the longer distance between the proton and carbonyl carbon. Furthermore, the signal intensities of the TO-DP were the same as that of the TO-CNF. This result indicates that the mobility of the carbonyl carbon on the surface of cellulose crystallite is independent of the crystal size.

Figure 3c shows the change in the NMR spectra at $2 \mathrm{~ms}$ $\mathrm{CT}$ before and after TEMPO-oxidation. The signals corresponding to $\mathrm{C}=\mathrm{O}$, trans-gauche $(t g)$, gauche-trans $(g t)$, and gauche-gauche $(g g)$ are centered at approximately $174.8,65.2,62.6$, and $60.6 \mathrm{ppm}$, respectively [28]. By the oxidation, the signal corresponding to the noncrystalline $g g$ or $g t$ was remarkably decreased, whereas the signal of the crystalline $\operatorname{tg}$ ratio remained constant, and the signal of the carbonyl carbon appeared at approximately 175 ppm. As shown in Fig. 3d, there was a 19\% decrease in the signal ratio of the noncrystalline hydroxy group, and this value is in good agreement with the increase in the signal of the carbonyl carbon (22\%). This result is consistent with data reported by Montanari et al. [29], suggesting the conversion to carboxyl groups from the hydroxy groups can be evaluated from the NMR spectra under optimized conditions. Furthermore, the relationship between the CI value and the degree of oxidation of the samples, see Fig. 3e, shows that the degree of oxidation can be evaluated from the NMR spectra at the optimized CT, irrespective of the crystallinity of cellulose. Note here that the total C6 signal intensities were slightly smaller than the $\mathrm{C} 1$ signal intensity, and the degree of oxidation evaluated from the spectrum was lower by 
approximately $16 \%$ than that estimated from conductivity titrations (Fig. 3e). This is probably because the chemical structure and molecular mobility are different between the C6 noncrystalline carbons and C1 carbons [15, 30], and both the total C6 signal and the amount of the carboxy groups were underestimated in the present pulse condition.

\section{Relationship between C4-based Cl value and C6-based tg ratio}

Based on a computational study, it has been reported that the conformation of the exocyclic groups at C6 significantly influences the $\mathrm{C} 4$ peak separation, and the signal at approximately $89 \mathrm{ppm}$, corresponding to the crystalline $\mathrm{C} 4$ carbon, is dominated by the $t g$ conformation [31]. Figure 4 shows the relationship between the C4-based $\mathrm{CI}$ value and the C6-based $t g$ ratio of samples with different crystal sizes. Interestingly, the CI value was found to be linearly correlated to the $t g$ ratio, with a slope of $0.9\left(R^{2}=0.98\right)$. This result demonstrates that the change in the $\mathrm{C} 4$ crystalline signal is strongly affected by the $t g$ conformation. The slight difference from 1.0 in the slope leads to the interpretation that the glycosidic linkage is partly restricted even when the C6 hydroxy groups sit in the noncrystalline $g t$ conformation [31].

\section{Conclusion}

We investigated the CP dynamics of cellulose with different crystal sizes for evaluation of the $\mathrm{CI}$ value. The error in the CI at the optimized CT of $2 \mathrm{~ms}$ was $\sim 3 \%$. In addition, at the optimized $\mathrm{CT}$, the structural change

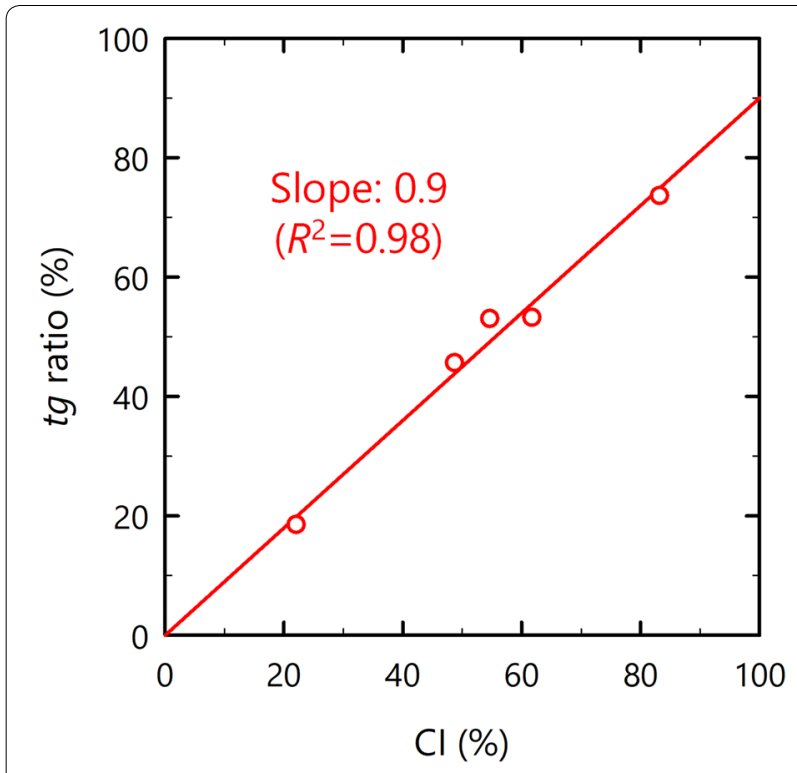

Fig. 4 Relationship between the $\mathrm{Cl}$ value and tg ratio by TEMPO-oxidation was also analyzed from the NMR spectra of the C6 region. Although the signal ratio of the noncrystalline C6 carbon is slightly underestimated, it is possible to evaluate the molecular dynamics and the degree of oxidation from the NMR spectra, irrespective of the crystallinity of cellulose. Furthermore, the correlation between the C4-based CI value and the C6-based tg ratio was investigated, and the C4-based CI value was linearly correlated with the C6-based $t g$ ratio, which is approximated by a function $(\mathrm{CI}=0.9 \mathrm{tg}$ ratio).

Importantly, we demonstrated that the $\mathrm{CI}$ value and the structural change of cellulose with different crystal sizes can be semiquantitatively evaluated by using NMR under optimized CP conditions.

\section{Abbreviations}

CP MAS NMR: Cross-polarization/magic-angle spinning nuclear magnetic resonance; Cl: Crystallinity index; CT: Contact time; DP: Dissolving pulp; TO-DP: TEMPO-oxidized DP; TO-CNF: TEMPO-oxidized cellulose nanofiber; XRD: X-ray diffraction; tg: Trans-gauche; gt: Gauche-trans; gg: Gauche-gauche.

\section{Acknowledgements}

We acknowledge Dr. Kazuo Furihata for arranging the NMR measurement.

\section{Authors' contributions}

KD, TS and SF conceived the concept of the study. All the authors designed the experiments. KD performed all the experiments. All the authors analyzed the data. KD and SF mainly wrote the manuscript with contributions from all the authors. All authors read and approved the final manuscript.

\section{Funding}

This research was in part supported by the JST-Mirai R\&D Program (JPMJMI17ED), JSPS Grant-in-Aids for Young Scientists (17K15298; 18K14501), JSPS Grants-in-Aid for Scientific Research (Grant number 20J12793), and a PHOENIX Grant-in-Aid (K. Daicho, 2019).

\section{Availability of data and materials}

The datasets generated during and/or analyzed during the current study are available from the corresponding author on reasonable request.

\section{Competing interests}

The authors declare that they have no competing interests.

\section{Author details}

${ }^{1}$ Graduate School of Agricultural and Life Sciences, The University of Tokyo, Bunkyo-ku, Tokyo 113-8657, Japan. ${ }^{2}$ Division of Forest and Biomaterials Science, Graduate School of Agriculture, Kyoto University, Sakyo-ku, Kyoto 606-8502, Japan. ${ }^{3}$ JEOL Ltd, Chiyoda-ku, Tokyo 100-0004, Japan.

Received: 8 May 2020 Accepted: 1 September 2020

Published online: 14 September 2020

\section{References}

1. Saito T, Kuramae R, Wohlert J, Berglund LA, Isogai A (2013) An ultrastrong nanofibrillar biomaterial: the strength of single cellulose nanofibrils revealed via sonication-induced fragmentation. Biomacromol 14(1):248-253

2. Uetani K, Okada T, Oyama HT (2015) Crystallite size effect on thermal conductive properties of nonwoven nanocellulose sheets. Biomacromol 16(7):2220-2227

3. Inui T, Koga H, Nogi M, Komoda N, Suganuma K (2015) A miniaturized flexible antenna printed on a high dielectric constant nanopaper composite. Adv Mater 27(6):1112-1116 
4. Saito T, Nishiyama Y, Putaux JL, Vignon M, Isogai A (2006) Homogeneous suspensions of individualized microfibrils from TEMPO-catalyzed oxidation of native cellulose. Biomacromol 7(6):1687-1691

5. Noguchi Y, Homma I, Matsubara Y (2017) Complete nanofibrillation of cellulose prepared by phosphorylation. Cellulose 24(3):1295-1305

6. Yang X, Reid MS, Olsen P, Berglund LA (2020) Eco-friendly cellulose nanofibrils designed by nature: effects from preserving native state. ACS Nano 14(1):724-735

7. Horii F, Hirai A, Kitamaru R (1982) Solid-state high-resolution 13C-NMR studies of regenerated cellulose samples with different crystallinities. Polym Bullet 8(2-4):163

8. Atalla RH, Vanderhart DL (1984) Native cellulose: a composite of two distinct crystalline forms. Science 223(4633):283-285

9. Newman $\mathrm{RH}$, Hemmingson JA (1990) Determination of the degree of cellulose crystallinity in wood by carbon-13 nuclear magnetic resonance spectroscopy. Holzforschung 44(5):351-356

10. Kono H, Yunoki S, Shikano T, Fujiwara M, Erata T, Takai M (2002) CP/MAS 13C NMR study of cellulose and cellulose derivatives. 1. Complete assignment of the CP/MAS 13C NMR spectrum of the native cellulose. J Am Chem Soc 124(25):7506-7511

11. Vietor RJ, Newman RH, Ha MA, Apperley DC, Jarvis MC (2002) Conformational features of crystal-surface cellulose from higher plants. Plant J 30(6):721-731

12. Phyo $P$, Wang T, Yang $Y, O^{\prime}$ Neill H, Hong M (2018) Direct determination of hydroxymethyl conformations of plant cell wall cellulose using (1)H polarization transfer solid-state NMR. Biomacromol 19(5):1485-1497

13. Earl WL, VanderHart DL (1981) Observations by high-resolution carbon-13 nuclear magnetic resonance of cellulose I related to morphology and crystal structure. Macromolecules 14(3):570-574

14. Larsson PT, Wickholm K, Iversen T (1997) A CP/MAS13C NMR investigation of molecular ordering in celluloses. Carbohyd Res 302(1-2):19-25

15. Hill DJT, Le TT, Whittaker AK (1994) A technique for the quantitative measurements of signal intensities in cellulose-based transformer insulators by13C CPMAS NMR. Cellulose 1(4):237-247

16. Heux L, Dinand E, Vignon MR (1999) Structural aspects in ultrathin cellulose microfibrils followed by 13C CP-MAS NMR. Carbohyd Polym 40(2):115-124

17. Daicho K, Kobayashi K, Fujisawa S, Saito T (2020) Crystallinity-independent yet modification-dependent true density of nanocellulose. Biomacromol 21(2):939-945

18. Daicho K, Saito T, Fujisawa S, Isogai A (2018) The Crystallinity of nanocellulose: dispersion-induced disordering of the grain boundary in biologically structured cellulose. ACS Appl Nano Mater 1(10):5774-5785

19. Okita $Y$, Saito T, Isogai $A$ (2010) Entire surface oxidation of various cellulose microfibrils by TEMPO-mediated oxidation. Biomacromol 11(6):1696-1700
20. Nemoto J, Saito T, Isogai A (2015) Simple freeze-drying procedure for producing nanocellulose aerogel-containing, high-performance air filters. ACS Appl Mater Interfaces 7(35):19809-19815

21. Terenzi C, Prakobna K, Berglund LA, Furo I (2015) Nanostructural effects on polymer and water dynamics in cellulose biocomposites: (2)h and (13) c NMR relaxometry. Biomacromol 16(5):1506-1515

22. Sparrman T, Svenningsson L, Sahlin-Sjovold K, Nordstierna L, Westman G, Bernin D (2019) A revised solid-state NMR method to assess the crystallinity of cellulose. Cellulose 26(17):8993-9003

23. Funahashi R, Okita Y, Hondo H, Zhao M, Saito T, Isogai A (2017) Different conformations of surface cellulose molecules in native cellulose microfibrils revealed by layer-by-layer peeling. Biomacromol 18(11):3687-3694

24. Oehme DP, Downton MT, Doblin MS, Wagner J, Gidley MJ, Bacic A (2015) Unique aspects of the structure and dynamics of elementary Ibeta cellulose microfibrils revealed by computational simulations. Plant Physiol 168(1):3-17

25. Wickholm K, Larsson PT, Iversen T (1998) Assignment of non-crystalline forms in cellulose I by CP/MAS 13C NMR spectroscopy. Carbohyd Res 312(3):123-129

26. Isogai A, Atalla RH (1991) Amorphous celluloses stable in aqueous media: regeneration from $\mathrm{SO}_{2}$-amine solvent systems. J Polym Sci 29(1):113-119

27. Saito T, Hirota M, Tamura N, Kimura S, Fukuzumi H, Heux L, Isogai A (2009) Individualization of nano-sized plant cellulose fibrils by direct surface carboxylation using TEMPO catalyst under neutral conditions. Biomacromol 10(7):1992-1996

28. Horii F, Hirai A, Kitamaru R (1983) Solid-state 13C-NMR study of conformations of oligosaccharides and cellulose. Polym Bull 10(7-8):357-361

29. Montanari S, Roumani M, Heux L, Vignon MR (2005) Topochemistry of carboxylated cellulose nanocrystals resulting from TEMPO-mediated oxidation. Macromolecules 38(5):1665-1671

30. Horii F, Hirai A, Kitamaru R (2006) CP/MAS Carbon-13 NMR study of spin relaxation phenomena of cellulose containing crystalline and noncrystalline components. J Carbohydr Chem 3(4):641-662

31. Yang H, Wang T, Oehme D, Petridis L, Hong M, Kubicki JD (2017) Structura factors affecting 13C NMR chemical shifts of cellulose: a computational study. Cellulose 25(1):23-36

\section{Publisher's Note}

Springer Nature remains neutral with regard to jurisdictional claims in published maps and institutional affiliations.

\section{Submit your manuscript to a SpringerOpen ${ }^{\circ}$ journal and benefit from:}

- Convenient online submission

- Rigorous peer review

- Open access: articles freely available online

- High visibility within the field

- Retaining the copyright to your article

Submit your next manuscript at $>$ springeropen.com 